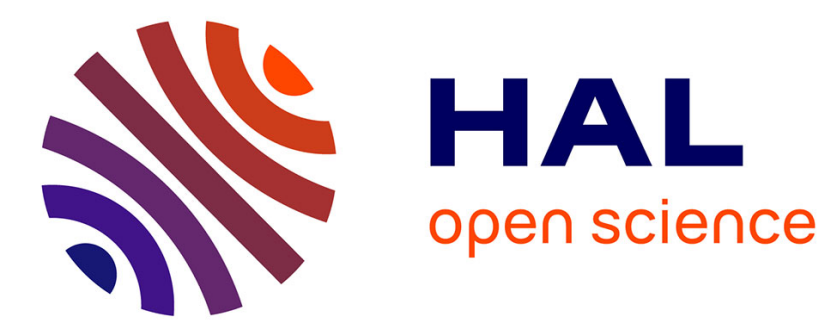

\title{
Synthesis and Simulation of Ensembles of Boolean Networks for Cell Fate Decision
}

Stéphanie Chevalier, Vincent Noël, Laurence Calzone, Andrei Zinovyev, Loïc Paulevé

\section{- To cite this version:}

Stéphanie Chevalier, Vincent Noël, Laurence Calzone, Andrei Zinovyev, Loïc Paulevé. Synthesis and Simulation of Ensembles of Boolean Networks for Cell Fate Decision. 18th International Conference on Computational Methods in Systems Biology (CMSB), 2020, Online, Germany. pp.193-209, 10.1007/978-3-030-60327-4_11. hal-02898849v2

\section{HAL Id: hal-02898849}

\section{https://hal.science/hal-02898849v2}

Submitted on 21 Aug 2020

HAL is a multi-disciplinary open access archive for the deposit and dissemination of scientific research documents, whether they are published or not. The documents may come from teaching and research institutions in France or abroad, or from public or private research centers.
L'archive ouverte pluridisciplinaire HAL, est destinée au dépôt et à la diffusion de documents scientifiques de niveau recherche, publiés ou non, émanant des établissements d'enseignement et de recherche français ou étrangers, des laboratoires publics ou privés. 


\title{
Synthesis and Simulation of Ensembles of Boolean Networks for Cell Fate Decision
}

\author{
Stéphanie Chevalier ${ }^{\star 1}$, Vincent Noë ${ }^{\star 2}$, Laurence Calzone ${ }^{2}$, Andrei Zinovyev ${ }^{2,3}$, \\ and Loïc Paulevé ${ }^{4}$ \\ 1 LRI, CNRS, UMR8623, Univ. Paris-Saclay, France \\ 2 Institut Curie, INSERM, U. PSL, Mines ParisTech, France \\ 3 Lobachevsky University, 603000 Nizhny Novgorod, Russia \\ ${ }^{4}$ Univ. Bordeaux, Bordeaux INP, CNRS, LaBRI, UMR5800, F-33400 Talence, France
}

\begin{abstract}
The construction of models of biological networks from prior knowledge and experimental data often leads to a multitude of candidate models. Devising a single model from them can require arbitrary choices, which may lead to strong biases in subsequent predictions.

We introduce here a methodology for a) synthesizing Boolean model ensembles satisfying a set of biologically relevant constraints and b) reasoning on the dynamics of the ensembles of models. The synthesis is performed using Answer-Set Programming, extending prior work to account for solution diversity and universal constraints on reachable fixed points, enabling an accurate specification of desired dynamics. The sampled models are then simulated and the results are aggregated through averaging or can be analyzed as a multi-dimensional distribution.

We illustrate our approach on a previously published Boolean model of a molecular network regulating the cell fate decisions in cancer progression. It appears that the ensemble-based approach to Boolean modelling brings new insights on the variability of synergistic interacting mutations effect concerning propensity of a cancer cell to metastasize.
\end{abstract}

\section{Introduction}

The ability to derive one single model from observations of a biological system usually faces arbitrary choices, sometimes referred to as art.

Computational models of molecular interaction networks are usually built from data related to the architecture of the network from known interactions; and data related to its dynamics, such as measurements of gene expressions or proteins activity at different times and/or conditions. However, despite huge advances in experimental technologies, observations of the biological processes stay very scarce, either in terms of temporal resolution, number of observed entities, synchronisation between measure points, or a variety of experimental conditions. Combined with complex structures for molecular interactions, the model engineering problem, in this case, appears to be largely under-specified, leading to (too) many potential candidate models.

\footnotetext{
* co-first authors
} 
Boolean Networks (BNs), and logical models in general, are widely adopted for the modelling of signalling pathways and gene and transcription factors networks 5283 . With BNs, the activity of components is caricatured to "off" and "on", and their evolution is computed according to logical rules (e.g., gene 1 can be active only whenever its activators 2 and 3 are active). However, in practice, biological data still let open a multitude of candidate BNs. Thus, arbitrary modelling choices have to be made, e.g., by prioritizing certain logics between regulators or by preferring smallest/largest models, which may introduce biases in subsequent model predictions.

In this paper, we present an approach aiming at reducing modelling biases by constructing and reasoning on dynamics of ensembles of BNs. The idea of ensemble modelling has recently gained momentum with machine learning, notably with random forests. By analogy, we constitute ensembles of BNs sampled from the whole multitude of models compatible with network architecture and dynamical properties. They are then simulated asynchronously and the simulations are aggregated through averaging. The obtained results allow an interpretation at the level of cell population and take into account its potential heterogeneity.

In the literature, ensembles of random BNs have been employed to show emerging properties of families of BNs sharing properties related to their architecture or logic rules [1416. In 21, ensembles of BNs sharing a network architecture are used to assess dynamical properties of qualitative differential equations. In contrast, our approach is focused on ensembles of models which satisfy a set of constraints both on their architecture and on their dynamics. Synthesis of BNs from such constraints received a lot of interest in the literature 6 24/7/27/32], and methods like 6 6 27/132 allows reasoning implicitly on ensembles of models, notably by enabling checking for their emptiness.

Here, we extend prior work on BNs synthesis from reachability and attractor properties with Most Permissive semantics 2] to support universal properties on (reachable) fixed points and the specification of network perturbations. The synthesis is performed with the logic framework of Answer-Set Programming [1] (ASP). Then, we use heuristics to drive the ASP solver in different regions of the solution space to sample ensembles of BNs capturing the diversity of the comprehensive solution set. Dynamics of ensembles are then explored through stochastic simulations for quantifying the propensities of reachable attractors, subject to different network perturbations. To that aim, we extended the simulator MaBoSS 22] to support ensembles of BNs as input.

We illustrate our approach on a model of molecular pathways regulating tumour invasion and migration [4]. We sampled ensembles of BNs sharing the same network architecture as the original model and constrained by the dynamical properties related to attractor reachability. Then, as in the original study, we evaluated the shift of reachable phenotypes caused by an epistatic interaction between mutations in model genes (gain of Notch function and loss of p53 function). It appears that, contrary to the initial single model analysis, the ensemble approach reveals a potential variability in the effectiveness of the double mutant to enhance the metastasis potential. 


\section{Background}

\subsection{Boolean Networks}

A Boolean network $(\mathrm{BN})$ of dimension $n$ is a function

$$
f: \mathbb{B}^{n} \rightarrow \mathbb{B}^{n}
$$

where $\mathbb{B}:=\{0,1\}$. For all $i \in[n], f_{i}: \mathbb{B}^{n} \rightarrow \mathbb{B}$ denotes the local function of the $i$-th component. A vector $x \in \mathbb{B}^{n}$ is called a configuration of the BN $f$. The set of components which value differs between two configurations $x, y \in \mathbb{B}^{n}$ is denoted by $\Delta(x, y):=\left\{i \in[n] \mid x_{i} \neq y_{i}\right\}$.

A BN $f$ is said locally monotonic whenever each of its local functions is monotonic (this does not imply $f$ monotonicity). Intuitively, local monotonicity imposes that a variable always appears with the same sign in a minimal disjunctive/conjunctive normal form of the local functions.

Fig. 1 is an example of locally-monotonic BN with $n=3$.

Mutations In the following, we will consider the analysis of a $\mathrm{BN} f$ subject to some permanent perturbations of its components, that we refer to as mutations, being either a gain of function (GoF; locked to 1) and loss of function (LoF; locked to 0$)$. A mutation is specified by a couple $(i, v)$, where $i \in[n]$ is a component and $v \in \mathbb{B}$ is its forced value. Given a BN $f$ and a set of mutations $M \subseteq[n] \times \mathbb{B}$, we denote by $f / M$ the mutated BN, where, for each $i \in[n]$, $(f / M)_{i}(x):=v$ if $(i, v) \in M$, and $(f / M)_{i}(x):=f_{i}(x)$ otherwise.

Influence graph For each $i \in[n], f_{i}$ typically depends on a small subset of components of the BN. The influence graph summarizes these dependencies with a positive (resp. negative) edge from node $j$ to $i$ if there are configurations in which the sole increase of $j$ would strictly increase (resp. decrease) the value of $f_{i}$. A node can have both positive and negative influences on $i$, indicating that $f_{i}$ is non-monotonic. Remark that different BNs can have the same influence graph. Fig. 1 (right) shows the influence graph of the BN example.

Definition 1. Given a $B N f$ of dimension $n$, its influence graph $G(f)$ is a directed graph $\left([n], E_{+}, E_{-}\right)$with positive and negative edges such that $(j, i) \in$ $E_{+}$(resp. $\left.(j, i) \in E_{-}\right)$iff $\exists x, y \in \mathbb{B}^{n}$ s.t. $\Delta(x, y)=\{j\}, x_{j}<y_{j}$, and $f_{i}(x)<$ $f_{i}(y)$ (resp. $\left.f_{i}(x)>f_{i}(y)\right)$. The influence graph $\mathcal{G}=\left([n], E_{+}, E_{-}\right)$is a subgraph of $\mathcal{G}^{\prime}=\left([n], E_{+}^{\prime}, E_{-}^{\prime}\right)$, denoted by $\mathcal{G} \subseteq \mathcal{G}^{\prime}$, iff $E_{+} \subseteq E_{+}^{\prime}$ and $E_{-} \subseteq E_{-}^{\prime}$.

$$
\begin{aligned}
& f_{1}(x):=\neg x_{2} \\
& f_{2}(x):=\neg x_{1} \\
& f_{3}(x):=\neg x_{1} \wedge x_{2}
\end{aligned}
$$

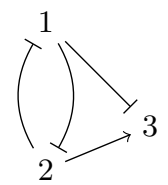

Fig. 1. Example of Boolean network $f$ and its influence graph $G(f)$ where positive edges are with normal tip and negative edges are with bar tip. 


\section{$2.2 \quad$ BN Semantics}

From a configuration $x \in \mathbb{B}^{n}$, semantics of BNs specify how to compute the next possible configurations. One of the most classical semantics is the fullyasynchronous (often simply called asynchronous), where only one component $i$ is updated at a time (to the value $f_{i}(x)$ ). It can be defined as a binary relation $\underset{\text { a1 }}{\stackrel{f}{\longrightarrow}}$ between configurations:

\section{Definition 2 (Fully-asynchronous semantics).}

$$
\forall x, y \in \mathbb{B}^{n}, \quad x \underset{\mathrm{a} 1}{\stackrel{f}{\longrightarrow}} y \text { iff } \exists i \in[n]: \Delta(x, y)=\{i\} \wedge y_{i}=f_{i}(x) .
$$

We write $\rho_{\mathrm{a} 1}^{f}(x):=\left\{y \in \mathbb{B}^{n} \mid x{\underset{\mathrm{a} 1}{\mathrm{f}}}^{*} y\right\}$ the set of configurations in transitive relation with $x$, with $\underset{\mathrm{a} 1}{\stackrel{f}{\longrightarrow}} *$ the reflexive and transitive closure of $\underset{\mathrm{a} 1}{\mathrm{f}}$.

However, as demonstrated in [19], the fully-asynchronous semantics of BNs, as the synchronous and (general) asynchronous, are not faithful abstractions of quantitative systems: they can both introduce spurious behaviours (as expected with qualitative models) and miss others.

The Most Permissive (MP) semantics of BNs [19] offers the guarantees to not preclude any behaviour realisable in any quantitative refinement of the model, thus providing a formal over-approximation of dynamics. Moreover, the abstraction is minimal: any behaviour it predicts is realisable by a quantitative refinement of the $\mathrm{BN}$ using the asynchronous semantics. Importantly, the complexity for deciding main dynamical properties is considerably lower than with (a)synchronous semantics, as we will mention in the next subsection.

MPBNs can be defined by the means of hypercubes (partially) closed by $f$, a hypercube being specified by a vector associating each component to either a fixed Boolean value or free $(*)$.

Definition 3 (Hypercube). A hypercube $h$ of dimension $n$ is a vector in $(\mathbb{B} \cup\{*\})^{n}$. The set of its associated configurations is denoted by $c(h):=\{x \in$ $\left.\mathbb{B}^{n} \mid \forall i \in[n], h_{i} \neq * \Rightarrow x_{i}=h_{i}\right\}$.

Given two hypercubes $h, h^{\prime} \in(\mathbb{B} \cup\{*\})^{n}, h$ is smaller than $h^{\prime}$ if and only if $\forall i \in[n], h_{i}^{\prime} \neq * \Rightarrow h_{i}=h_{i}^{\prime}$.

Definition 4 (K-closed hypercube). Given a subset of components $K \subseteq[n]$, a hypercube $h \in(\mathbb{B} \cup\{*\})^{n}$ is $K$-closed by $f$ whenever for each configuration $x \in c(h)$, for each component $i \in K, h_{i} \in\left\{*, f_{i}(x)\right\}$.

It is minimal whenever no different $K$-closed hypercube is smaller than it.

A hypercube $[n]$-closed by $f$ is also known as a trap space [15].

Example 1. Let us consider the BN $f: \mathbb{B}^{3} \rightarrow \mathbb{B}^{3}$ with $f_{1}(x):=1, f_{2}(x):=x_{1}$, and $f_{3}(x):=x_{1} \wedge \neg x_{3}$. The hypercube $1 * *$ is closed by $f$, with $c(1 * *)=$ $\{100,101,110,111\}$. The hypercube $1 * 0$ is the smallest hypercube $\{2\}$-closed by $f$ containing 100 ; it is not closed by $f$, nor the smallest hypercube $\{2\}$-closed by $f$ containing 110 . 

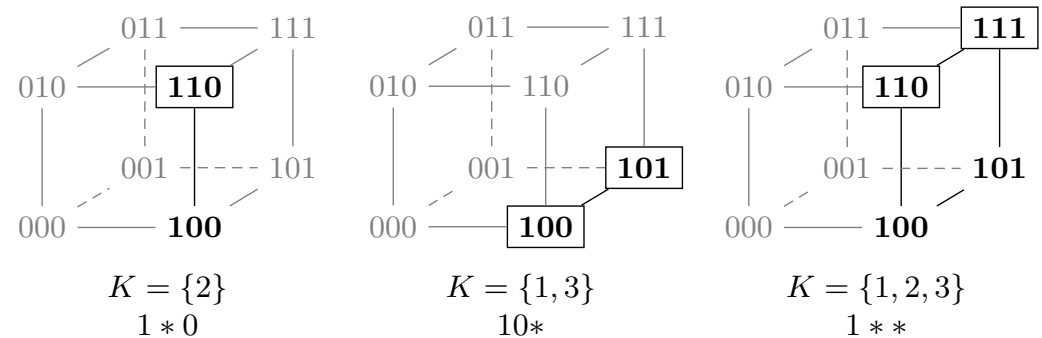

Fig. 2. Examples of smallest $K$-closed hypercubes containing the configuration 100 for the BN $f$ of dimension 3 defined by $f_{1}(x):=1, f_{2}(x):=x_{1}, f_{3}(x):=x_{1} \wedge \neg x_{3}$. Configurations belonging to the hypercube are in bold; these verifying the MP reachability property are boxed. The hypercube $11 *$ is the only one which is closed by $f$ and minimal.

Starting from a configuration $x \in \mathbb{B}^{n}$, the MP semantics allows transitions towards any configuration $y$ which is present in at least one smallest $K$-closed hypercube $h$ containing $x$, for some $K \subseteq n$, and so that the state of each component $i \in K$ of $y$ can be computed by $f_{i}$ from a configuration of $h$.

Definition 5 (Most-Permissive semantics). Given a $B N f$ of dimension $n$ and two configurations $x, y \in \mathbb{B}^{n}, y \in \rho_{\mathrm{mp}}^{f}(x)$ if and only if there exists $K \subseteq[n]$ such that the smallest $K$-closed hypercube $h$ containing $x$ verifies (1) $y \in c(h)$, and (2) $\forall i \in K$, there exists a configuration $z \in c(h)$ such that $f_{i}(z)=y_{i}$.

Fig. 2 gives examples of computations of $\rho_{\mathrm{mp}}^{f}$.

A way to interpret the MP semantics is to see the components free in a hypercube as being in the course of changing of state, while other components can independently consider them either as 0 or 1 . This abstracts the missing information on the ordering of thresholds of activation/inhibition between components: while the quantitative value of component $u$ progressively increases, at a given time it can be high enough to activate a component (i.e., 1) but not yet high enough to activate another one (i.e., 0). These dynamic states are overlooked by asynchronous semantics, making it an incorrect over-approximation of quantitative systems, contrary to the MP semantics [19].

\subsection{Dynamical properties}

In the following, we will focus on two main dynamical properties of BNs: reachability which relates to the existence of trajectories between two configurations, and attractors which relates to long-run behaviours by identifying the smallest sets of configurations closed by reachability.

Definition 6 (Reachability). Given two configurations $x, y \in \mathbb{B}^{n}$ of a $B N f$ with semantics $\sigma, y$ is reachable from $x$ whenever $y \in \rho_{\sigma}^{f}(x)$. 
Definition 7 (Attractor). A non-empty set of configurations $A \subseteq \mathbb{B}^{n}$ is an attractor of the $B N f$ with semantics $\sigma$ whenever $\forall x \in A, \rho_{\sigma}^{f}(x)=A$. When $A=\{x\}$ for some $x \in \mathbb{B}^{n}$, we say that $x$ is a fixed point.

With MP semantics, attractors match with minimal trap spaces. With fullyasynchronous semantics, deciding if $y \in \rho_{\mathrm{a} 1}^{f}(x)$ or if $x$ belongs to an attractor are both PSPACE-complete problems. With MP semantics, deciding if $y \in \rho_{\mathrm{mp}}^{f}(x)$ is PTIME if $f$ is locally-monotonic and $\mathrm{P}^{\mathrm{NP}}$ otherwise; deciding if $x$ belongs to an attractor is coNP if $f$ is locally-monotonic and coNPcoNP otherwise. Deciding if there exists a fixed point is NP-complete with both semantics [19.

Notice the following relations between MP and fully-asynchronous semantics:

- $x \in \mathbb{B}^{n}$ is a fixed point with MP semantics if and only if it is a fixed point with full-asynchronous semantics (iff $f(x)=x$ );

- $y \in \mathbb{B}^{n}$ is reachable from $x \in \mathbb{B}^{n}$ with the fully-asynchronous semantics only if it is reachable with MP semantics $\left(\rho_{\mathrm{a} 1}^{f}(x) \subseteq \rho_{\mathrm{mp}}^{f}(x)\right)$;

- the number of attractors with MP semantics is less than or equal to the number of attractors with fully-asynchronous semantics.

\subsection{Answer-Set Programming}

Answer Set Programming (ASP; [1]10]) is a declarative approach to solving combinatorial satisfaction problems. It is close to SAT (propositional satisfiability) [17] and known to be efficient for enumerating solutions of NP problems comprising up to tens of millions of variables while providing a convenient language for specifying the problem. We give a very brief overview of ASP syntax and semantics that we use in the next sections; see 10] for more details.

An ASP program is a Logic Program (LP) being a set of logical rules with first order logic predicates of the form:

${ }_{1} a_{0} \leftarrow a_{1}, \ldots, a_{n}, \operatorname{not} a_{n+1}, \ldots$, not $a_{n+k}$.

where $a_{i}$ are (variable-free) atoms, i.e., elements of the Herbrand base, which is built from all the possible predicates of the LP. The Herbrand base is built by instantiating the LP predicates with the LP terms (constants or elements of the Herbrand universe).

Essentially, such a logical rule states that when all $a_{1}, \ldots, a_{n}$ are true and none of $a_{n+1}, \ldots, a_{n+k}$ can be proven to be true, then $a_{0}$ has to be true as well. Whenever $a_{0}$ is $\perp$ (false), the rule, also called integrity constraint, becomes:

${ }_{2} \leftarrow a_{1}, \ldots, a_{n}$, not $a_{n+1}, \ldots$, not $a_{n+k}$.

Such a rule is satisfied only if the right-hand side of the rule is false (at least one of $a_{1}, \ldots, a_{n}$ is false or at least one of $a_{n+1}, \ldots, a_{n+k}$ is true). On the other hand, $a_{0} \leftarrow \top$ ( $a_{0}$ is always true) is abbreviated as $a_{0}$. A solution (answer set) is a stable Herbrand model, that is, a minimal set of true atoms where all the logical rules are satisfied. For instance, consider the following program: 


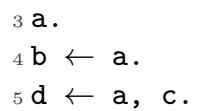

It has for unique solution $\{\mathrm{a}, \mathrm{b}\}$ : indeed, whereas $\{a, b, d\}$ does not contradict the rules, $\mathrm{d}$ is not a fact and cannot be derived from a rule; so it is not stable.

ASP allows using variables (starting with an upper-case) instead of terms/predicates: these template declarations will be expanded before the solving. We also use the notation $a(X): b(X)$ which is satisfied when for each $b(X)$ true, $a(X)$ is true. If any term follows such a condition, it is separated with ;

ASP can express disjunctive logic programs [18, by the means of disjunctions in the rule head (";"-separated atoms):

$6 \mathrm{a} ; \mathrm{b} \leftarrow$ body.

Such a disjunctive rule implies that solutions are subset minimal: an answer set is a solution only if none of its subsets is itself a solution [9]. For instance, let's consider the disjunction:

$7 \mathrm{a} ; \mathrm{b} ; \mathrm{c}$.

The interpretation $I=\{a, b\}$ is a model but not minimal: both interpretations $\{a\}$ and $\{b\}$ are smaller than $I$ and satisfy the rule. Hence $I$ is not a solution. As showed in [8, the complexity of problems addressed with ASP can be extended thanks to disjunctive rules up to $2 \mathrm{QBF}$, i.e. a two quantification levels Boolean formula ( $\forall x \exists y$. $\phi$ or $\exists y \forall x$. $\phi$ where $\phi$ is a quantifier-free propositional formula). Indeed, $2 \mathrm{QBF}$ can be reduced to the problem of verifying the existence of an answer set of a disjunctive ASP program.

\section{BN Synthesis from Architecture and Dynamical Properties}

We formulate the problem of BN synthesis as a Boolean satisfiability problem encoded in ASP. With this approach, we leverage a priori knowledge and experimental data as constraints on the network architecture and the dynamical properties of the models under the MP semantics. Our method is based on [2, which implements constraints on existence and absence of trajectories between partially-specified configurations, existence of (reachable) fixed points and trap spaces. In biological applications, these constraints match well the observed properties of cell populations evolving towards mutually exclusive phenotypes.

In this paper, we extend [2] to support universal properties on (reachable) attractors. This enables specifying tight dynamical constraints. For instance, given a set of experimentally observed phenotypes, existential constraints guarantee that at least one attractor of the model dynamics match with each phenotype, whereas a universal constraint ensures that every attractor matches with at least one of the phenotype.

A universal property involves by nature universal quantifiers. ASP can address formulas implying one level of universal quantifier (i.e., of the form $\exists x \forall y$ : 
$P(x, y))$ thanks to a technique presented in [8. To explore a set of values and check the respect of a property for each, it uses a disjunctive rule and a saturation on the same term. A disjunctive rule implies the subset minimality semantics. This minimality ensures an answer set is a solution only if none of its subsets is itself a solution [9]. Hence, saturating the answer set with the predicates of the disjunction cleverly exploits this minimality: the solver is forced to ensure that no strict subset of these predicates form a solution.

\subsection{Universal constraints on fixed points}

We exploit this saturation technique 89 for ensuring universal constraints on the fixed points or fixed points reachable from a given configuration. We describe here the ASP rules for the universal fixed point constraint, which ensures that all the fixed points of the BN are compatible with a given set of markers (observations). To that purpose, we let the solver deduce a configuration $z$ by the disjunctive rule:

$8 \operatorname{cfg}(z, N,-1) ; \operatorname{cfg}(z, N, 1) \leftarrow \operatorname{node}(N)$.

The predicate template $\mathrm{cfg}(\mathrm{X}, \mathrm{N}, \mathrm{V})$ assigns the value $\mathrm{V}$ to the literal $\mathrm{N}$ in the configuration $\mathrm{X}$. Through the above rule, a set of node values is thus constituted to define a configuration $z$, with the predicate $\operatorname{cfg}\left(z, \mathrm{~N}_{-}\right)$subject to the subset minimality semantics. To respect the desired property, each configuration $z$ is either not a fixed point $(f(z) \neq z)$ or has the same component states than the ones expressed in a dedicated predicate. A configuration is not a fixed point whenever at least one of its component can change of state:

$9 \operatorname{mcfg}(z, N, V) \leftarrow \operatorname{cfg}(z, N, V)$.

10 valid $\leftarrow \operatorname{cfg}(z, N, V) ; \operatorname{eval}(z, N,-V)$.

$\operatorname{mcfg}(\mathrm{X}, \mathrm{N}, \mathrm{V})$ predicate template leads to the evaluation of the configuration $\mathrm{X}$ given the Boolean rules of the network 2. The reachable values are then stored in the predicate eval $(\mathrm{X}, \mathrm{N}, \mathrm{V})$. Whenever it is possible to evaluate a component $\mathrm{N}$ to the opposite value than in $z$, then $z$ is not a fixed point, making valid true.

Otherwise, $z$ has to have the same component states than those specified by an observation $\mathrm{X}$ marked by the predicate is_universal_fp(X), which is expressed by the following ASP rule:

11 valid $\leftarrow \operatorname{cfg}(\mathrm{z}, \mathrm{N}, \mathrm{V}):$ obs $(\mathrm{X}, \mathrm{N}, \mathrm{V})$; is_universal_fp $(\mathrm{X})$.

Observe in 1,10 and 1,11 that each time an assignment is in agreement with the desired property, a predicate valid is deduced, which triggers the saturation of the configuration $z$ :

$12 \operatorname{cfg}(\mathrm{z}, \mathrm{N},-\mathrm{V}) \leftarrow \operatorname{cfg}(\mathrm{z}, \mathrm{N}, \mathrm{V})$, valid.

Thus, when valid is deduced, the answer set contains all possible component values for $z$. According to the subset minimality semantics, the solver is then forced to ensure that there is no sub-answer set. And the only way to find such a smaller answer-set is to find a $z$ from which valid cannot be deduced, i.e., which 
is a counter-example to the universal property: in that case, 1,13 eliminates the answer set:

$13 \leftarrow$ not valid.

A variant of this constraint enables to restrict the universal property to fixed points that are reachable from a given initial configuration. This is specified by is_universal_f $\mathrm{p}(\mathrm{X}, \mathrm{S})$ predicates, where $\mathrm{S}$ points the initial configuration, and $\mathrm{X}$ to an observation, as used above. By combining such predicates, one can then specify sets of phenotypes reachable from a given configuration. The encoding of this variant contains a third way to deduce valid: the non-reachability of the configuration $z$ from S.

Our implementation also offers to specify mutations, which can be combined with reachability and with universal constraints on reachable fixed points to leverage observations about cell fates in different mutation conditions.

\subsection{Synthesis problem}

Synthesis requires (i) an influence graph to delimit the interactions that can be used by the BNs and (ii) the dynamical properties of the behaviours that have to be reproduced. For modelling the tumour invasion and migration as in [4, the dynamical properties refer to cell fate observations in different mutation conditions. These fates are described by sets of markers (i.e. a set of values for some nodes of the network) which constitutes partial observations of genes activity. In term of dynamics, these observations are related to reachable attractors in the corresponding mutated BNs.

A (partial) observation $o$ of a configuration of dimension $n$ is specified by a set of couples associating a component to a Boolean value: $o \subseteq[1] n \times \mathbb{B}$, assuming there is no $i \in[n]$ such that $\{(i, 0),(i, 1)\} \subseteq 0$.

Formally, the synthesis problem we tackle is the following. Given

- an influence graph $\mathcal{G}=\left\{[n], E_{+}, E_{-}\right)$

- $p$ partial observations $o^{1}, \ldots, o^{p}$

- sets FP and UFP of indices of observations

- sets PR and URFP of couples of indices of observations: URFP $\subseteq[p]^{2}$

find a locally-monotonic $\mathrm{BN} f$ of dimension $n$ such that

$-G(f) \subseteq \mathcal{G}$,

- there exist $p$ configurations $x^{1}, \ldots, x^{p}$ such that:

- (observations) $\forall m \in[p], \forall(i, v) \in o^{m}, x_{i}^{m}=v$,

- (positive reachability) $\forall\left(m, m^{\prime}\right) \in \mathrm{PR}, x^{m^{\prime}} \in \rho_{\mathrm{mp}}^{f}\left(x^{m}\right)$,

- (fixed points) $\forall m \in \mathrm{FP}, f\left(x^{m}\right)=x^{m}$,

- (universal fixed point) $\forall z \in \mathbb{B}^{n}, f(z)=z \Rightarrow \exists m \in \mathrm{UFP}: \forall(i, v) \in o^{m}, z_{i}=v$;

- (universal reachable fixed point) $\forall\left(q, m^{\prime}\right) \in$ URFP, $\forall z \in \rho_{\mathrm{mp}}^{f}\left(x^{q}\right), z=f(z) \Rightarrow \exists(q, m) \in \operatorname{URFP}: \forall(i, v) \in o^{m}, z_{i}=v$. 
Each of these constraints can be parametrized by mutations, in which case, the properties have to be verified on the mutated $f$.

Remark that such a problem can be non-satisfiable.

Our encoding also offers constraints related to the absence of paths between configurations (negative reachability) and to trap space where a set of components have a fixed state matching with a given observation [2. Moreover, one can optionally impose that the influence graph of $f$ is equal to the input $\mathcal{G}$.

Our implementation avoids redundancy in the models by enumerating only among non-equivalent BNs (i.e., their values differ for at least one configuration). This is achieved by using a canonical representation of Boolean functions in disjunctive normal form with a total ordering between clauses.

In total, our encoding generates $O\left(n d k^{2}\right)$ atoms and $O\left(n d^{2} k^{2}\right)$ rules, where $d$ is the in-degree of nodes in the influence graph, and $k$ is a fixed bound on the number of clauses of Boolean functions. Whenever $k$ is set to $\left(\begin{array}{c}d \\ \lfloor d / 2\rfloor\end{array}\right)$, the complete set of solutions can be enumerated.

\subsection{Sampling the diversity of all solutions}

The whole set of constraints, comprising the domain of admissible BNs and the dynamical properties they should satisfy, is represented by a single logic program expressed in ASP, such that each solution corresponds to a distinct BN.

Whereas the enumeration of ASP solutions is known to be efficient, typical solvers will enumerate solutions by slightly varying parts of a firstly identified one. Thus, a partial enumeration will very likely give a set of solutions which are all look alike, e.g., where the Boolean function of only one component varies.

Inspired by [20], we tweak heuristics of the solver clingo [1] to stir it towards distant solutions: at each solution, we randomly select a subset of variables assignments and ask the solver to avoid them in the next iterations. At the cost of enumeration speed, this allows sampling ensembles of diverse BNs.

\section{Stochastic Simulations of Ensembles of BNs}

\subsection{Continuous-time Boolean modelling}

We first recall the continuous-time Markov chain interpretation of BNs introduced in 23. Considering a $\mathrm{BN} f$ of dimension of $n$, we represent the state evolution by a Markov process $s: t \rightarrow s(t)$ defined on $t \in I \subset \mathbb{R}$ applied on the network state space, with I the simulation interval. This process is defined by:

1. Its initial condition:

$$
P[s(0)=x], \quad \forall x \in \mathbb{B}^{n}
$$

2. Its conditional probabilities (of a single condition):

$$
P\left[s(t)=y \mid s\left(t^{\prime}\right)=x\right], \quad \forall x, y \in \mathbb{B}^{n}, \forall t^{\prime}, t \in I, t^{\prime}<t
$$


In continuous-time, these conditional probabilities are defined as transition rates [25]: $\rho(x \rightarrow y)(t) \in[0, \infty]$. Because we want a generalization of the fullyasynchronous Boolean dynamics, transition rates $\rho(x \rightarrow y)$ are non-zero only if $x \underset{\text { a1 }}{\stackrel{f}{\longrightarrow}} y$, i.e., a single component $i \in[n]$ is changing of value. In that case, each local function $f_{i}(x)$ is replaced by two functions $R_{i}^{u p / d o w n}(x) \in[0, \infty]$. The transition rates are defined as follows:

$$
\rho(x \rightarrow y)=\left\{\begin{array}{ll}
R_{i}^{u p}(x) & \text { if } x_{i}=0 \\
R_{i}^{\text {down }}(x) & \text { if } x_{i}=1
\end{array} \text { with } \Delta(x, y)=\{i\}\right.
$$

where $R_{i}^{u p}$ corresponds to the activation rate of node $i$, and $R_{i}^{\text {down }}$ corresponds to the inactivation rate of node $i$. Therefore, the continuous Markov process is completely defined by all these $R^{u p / d o w n}$ and an initial condition. By default, the value of these rates is set to 1 , but they can be modified to represent the time scales of different processes.

To explore the probability space of this Markov process, we use the Gillespie algorithm [12. This algorithm produces a set of realizations or stochastic trajectories of the Markov process. From this finite set, probabilities can be estimated.

To relate continuous-time probabilities to real processes, an observable time window $\delta t$ is defined. A discrete-time $\tau \in \mathbb{N}$ stochastic process $s(\tau)$ can be extracted from the continuous-time Markov process:

$$
P[s(\tau)=x] \equiv \frac{1}{\delta t} \int_{\tau \delta t}^{(\tau+1) \delta t} P[s(t)=x] d t
$$

For each trajectory $\mathrm{j}$, we compute the time for which the system is in state $\mathrm{x}$ in the window $[\tau \delta t,(\tau+1) \delta t]$, and divide it by $\delta t$. We obtain an estimate of $P[s(\tau)=x]$ for trajectory j, i.e. $\hat{P}_{j}[s(\tau)=x]$. Then to compute the estimate of a set of trajectories, we compute the average over $\mathrm{j}$ of all $\hat{P}_{j}[s(\tau)=x]$.

\subsection{Lifting to ensembles of $\mathrm{BNs}$}

To simulate an ensemble of BNs, we first choose a total number of stochastic trajectories $M$. We generate $M / k$ stochastic trajectories for each model and compute the average $\hat{P}_{k}[s(\tau)=x]$ for all models $k$. We then compute the average over k of all $\hat{P}_{k}[s(\tau)=x]$, to obtain the $P[s(\tau)=x]$ for the ensemble of boolean networks. We also keep the option to export the individual probability distributions $\hat{P}_{k}[s(\tau)=x]$ to allow us analyzing the composition of the ensemble. The approach results in time-series of the probability for each observed state. The case study hereafter focuses on steady-state analysis. This imposes to simulate the ensemble long enough to reach stationarity, requiring a preliminary analysis. We can then study the proportion of each attractor for our ensemble.

We implemented this new feature in the MaBOSS simulation software $[22]^{5}$

\footnotetext{
5 https://maboss.curie.fr https://github.com/colomoto/pyMaBoSS
} 


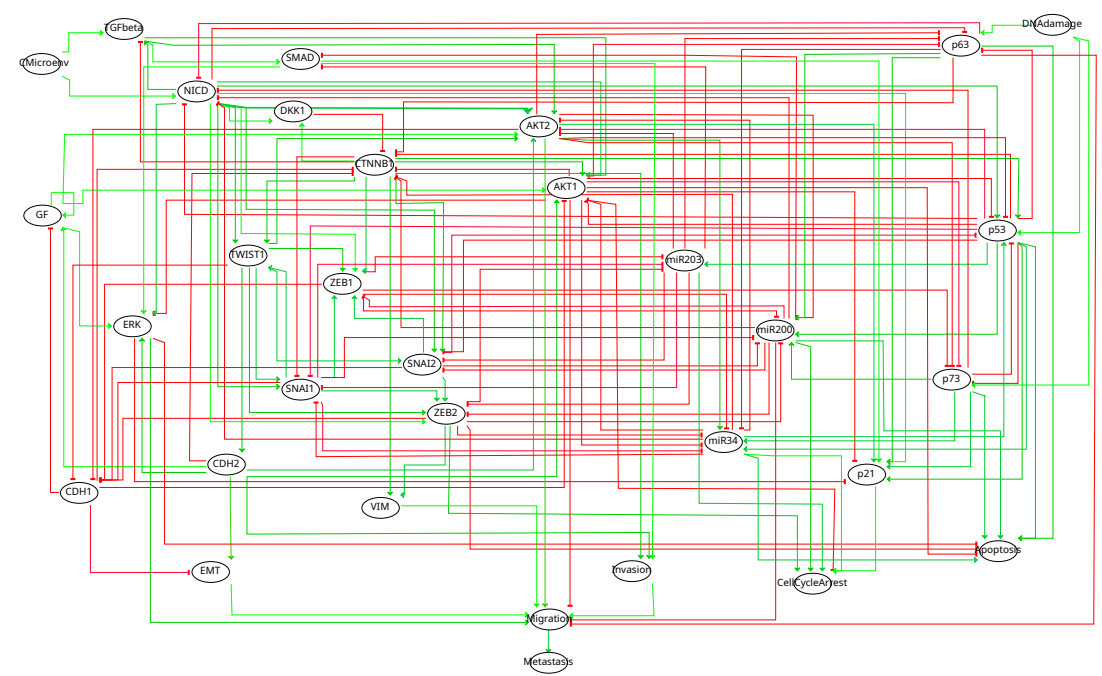

Fig. 3. Influence graph of Cohen's model relating 32 nodes with 159 edges, where positive edges are in green and negative in red.

\section{Case Study on Cell Fate Decision Modelling}

\subsection{Background Model}

We illustrate our ensemble modelling approach on a published model of cell fate decision leading to the early events of the metastasis or cell death through apoptosis 4 . Initial triggers, such as DNA damage or micro-environmental cues, and the activity of some genes or proteins participating in the process affect the final decision. The signalling pathway involves TGFbeta, WNT, beta-catenin, p53 and its homologs, selected miRNA, and transcription factors of the epithelial to mesenchymal (EMT) transitions. Fig. 3 shows the influence graph of the BN.

The functions of the BN, we refer to as "Cohen's model", have been designed manually so the simulations fit with experimental data related to stable phenotypes under different single mutations. Then, the initial publication explored the synergy between mutants that led to metastatic phenotypes.

\subsection{Single Model Analysis}

We first reproduced part of the analysis of [4] on the original Cohen's model by computing the propensities of attractors reachable from 4 possible initial conditions, where all nodes are inactive, except miRNAs that are active, and the 2 nodes modelling DNA damage and micro-environment cues that are free. We considered the wild-type condition (Fig. 4(a)) with no mutation, and the double-mutant of p53 LoF and Notch GoF (Fig. 4(b)). 
The wild-type model has 9 fixed points that each correspond to one of the 4 identified physiological phenotypes: Apoptosis, EMT, Metastasis (or equivalent to Migration) and Homeostatic State (HS). The double-mutant shows exclusively the Metastasis phenotype.

\subsection{Ensemble Analysis}

Synthesis To test the impact of alternative Boolean functions, we synthesised ensembles of BNs that share the same influence graph as Cohen's model and reproduce the desired dynamics. We synthesized two ensembles of 1,000 diverse BNs each, where we disallowed having cyclic attractors. The first ensemble ensures only the wild-type (WT) behaviour, meaning that all the fixed points match with one of the 4 physiological phenotypes, and each physiological phenotype is reachable from at least one of the initial condition. The second ensemble adds further constraints related to the single mutations of p53 LoF, which should show the same behaviour as WT, and Notch GoF, where only 2 of the WT phenotypes and a third different one should be observed 6 .

Ensemble Simulations With the same settings as with Cohen's model, we performed stochastic simulations of the two synthesized ensembles, with uniform activation and de-activation rates. The WT behaviours look similar, with some differences in propensities of phenotypes (Fig. 4(c,e)). The double-mutant on the ensembles shows a much less contrasted picture than on the Cohen's model. While Migration becomes the most likely outcome, several other phenotypes are observed, suggesting a potential variability of the effect of the double-mutation. Interestingly, even the single mutant constraints of the second ensemble are not sufficiently restrictive to guarantee the behaviour observed in Cohen's model.

Variability of propensities of phenotypes To study the ensemble composition, we want to analyze the steady-state probabilities for each model. Depending on the results we might have a lot of visited states, which bring a dimensionality issue. We choose to represent these results using Principal Component Analysis (PCA) 26], which allows us to visualize the distribution of attractor's proportions in a reduced number of dimensions.

We apply PCA to the probability distribution of each model within the ensemble from WT and single mutants constraints, allowing us to represent their respective probability distributions (Fig. 5). The first component, representing $56 \%$ of the observed variance, shows a negative correlation between apoptotic and EMT phenotypes. The second component, representing $24 \%$ of observed variance, shows a negative correlation between EMT without Migration and

\footnotetext{
${ }^{6}$ Code, data, and notebooks at https://doi.org/10.5281/zenodo.3938904, Synthesis has been performed on 36-cores CPUs @ 2.6Ghz with 192Go of RAM; first ensemble was generated at a rate of $5 \mathrm{~s} /$ model $/ \mathrm{CPU}$; second ensemble was generated at a rate of $3 \mathrm{~min} /$ model $/ \mathrm{CPU}$
} 


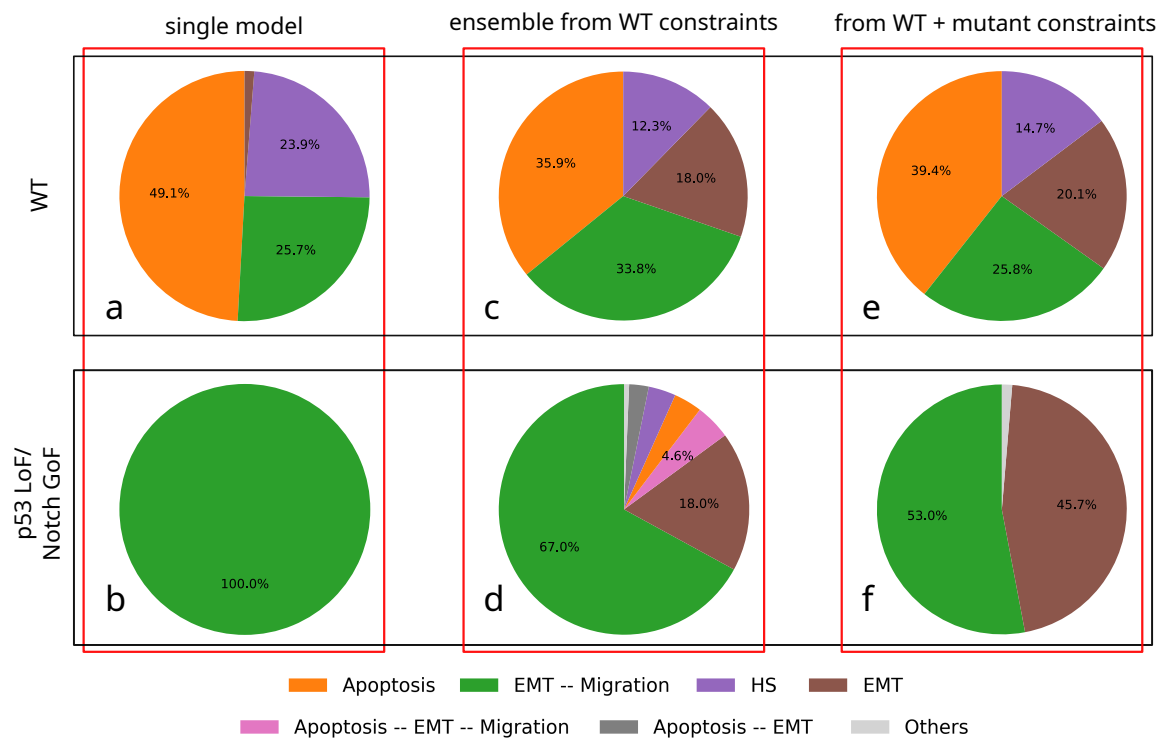

Fig. 4. Simulations results for phenotypes propensities in Cohen's model (a,b), ensemble from WT constraints $(\mathrm{c}, \mathrm{d})$, and ensemble from WT and single mutants constraints $(\mathrm{e}, \mathrm{f})$, in wild-type condition (a,c,e) and double-mutant p53 LoF/Notch GoF (b,d,f).

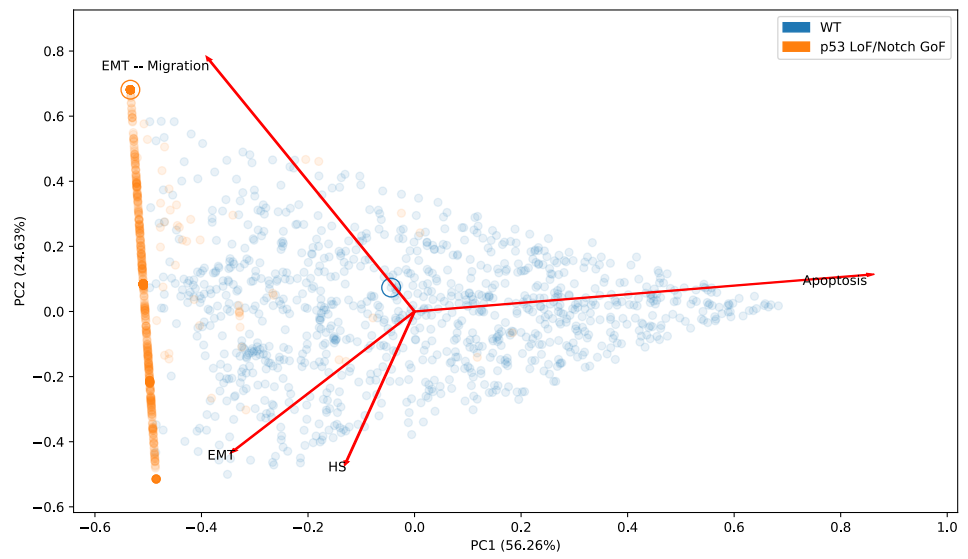

Fig. 5. PCA representation of the steady-state distribution of each model of the ensemble from WT and single mutants constraints. Each point represents the result of one model simulation (blue one are from WT simulations, orange one from p53 LoF/Notch GoF). Large blue and orange circles highlight the position of the original single Cohen's model simulation. The triangular pattern of the distribution comes from the fact that the phenotype probabilities are located in the $n$-dimensional simplex. 
EMT with Migration. The distribution of the ensemble's probability distributions is diverse, illustrating the performance of the all possible model diversity sampling. The p53 LoF/Notch GoF double mutant shows a shift towards EMT and/or Migration phenotypes, away from Apoptotic phenotypes. The alignment of models on the top-left corresponds to models which don't show any apoptotic phenotypes ( $\sim 96 \%$ of the models).

\section{Conclusion}

The synthesis of BNs from network architecture and dynamical constraints can lead to a multitude of admissible solutions.

In this work, we employed Answer-Set Programming to sample ensembles of diverse BNs, all possessing the same network architecture and satisfying the same set of dynamical constraints. We significantly extend the previously described methodology with the new type of biologically relevant universal constraints.

Our synthesis framework enables specifying existence and absence of reachability properties between (partial) configurations of the BN, existence of fixed points and cyclic attractors matching with observations, and universal properties on the fixed points and reachable fixed points; all these properties can be parametrized by mutation settings.

The dynamics of ensembles is explored by stochastic simulations using the new Ensemble MaBoSS simulator, which is introduced here for the first time. The ensemble-based simulations are used for computing and comparing propensities of reachable attractors under different mutations or their combinations. The result of an ensemble-based simulation represents a multidimensional distribution of the vectors of attractor probabilities, which can be aggregated by computing its mean point. Moreover, the multi-variate variance of the distribution can be explored, e.g. by applying the standard machine learning methods such as Principal Component Analysis, which can lead to the insights about the diversity of possible modelling scenarios compatible with available biological knowledge and the experimental data.

As illustrated on a biological case study, BN ensemble modelling brings an insight into the potential variability of predictions subject to model uncertainty.

In future work, we plan to address the evaluation of the diversity of sampled ensembles, with metrics helping estimate required sample size and compare sampling heuristics.

Acknowledgements This work has been partially supported by Agence Nationale de la Recherche in the program Investissements d'Avenir (project No. ANR-19- P3IA-0001; PRAIRIE 3IA Institute), by ANR-FNR project "AlgoReCell" (ANR-16-CE12-0034), by ITMO Cancer, and by the Ministry of Science and Higher Education of the Russian Federation (project No. 14.Y26.31.0022). Experiments were carried out using the PlaFRIM experimental testbed, supported by Inria, CNRS (LABRI and IMB), Université de Bordeaux, Bordeaux INP and Conseil Régional d'Aquitaine (see https://www.plafrim.fr). 


\section{References}

1. Baral, C.: Knowledge Representation, Reasoning and Declarative Problem Solving. Cambridge University Press (2003)

2. Chevalier, S., Froidevaux, C., Paulevé, L., Zinovyev, A.: Synthesis of Boolean Networks from Biological Dynamical Constraints using Answer-Set Programming. In: 2019 IEEE 31st International Conference on Tools with Artificial Intelligence (ICTAI). pp. 34-41 (2019). https://doi.org/10.1109/ICTAI.2019.00014

3. Clarke, M.A., Fisher, J.: Executable cancer models: successes and challenges. Nature Reviews Cancer (apr 2020). https://doi.org/10.1038/s41568-020-0258-x

4. Cohen, D.P.A., Martignetti, L., Robine, S., Barillot, E., Zinovyev, A., Calzone, L.: Mathematical modelling of molecular pathways enabling tumour cell invasion and migration. PLoS Comput Biol 11(11), e1004571 (2015). https://doi.org/10.1371/journal.pcbi.1004571

5. Collombet, S., van Oevelen, C., Sardina Ortega, J.L., Abou-Jaoudé, W., Di Stefano, B., Thomas-Chollier, M., Graf, T., Thieffry, D.: Logical modeling of lymphoid and myeloid cell specification and transdifferentiation. Proceedings of the National Academy of Sciences 114(23), 5792-5799 (2017). https://doi.org/10.1073/pnas.1610622114

6. Corblin, F., Tripodi, S., Fanchon, E., Ropers, D., Trilling, L.: A declarative constraint-based method for analyzing discrete genetic regulatory networks. Biosystems 98(2), 91-104 (nov 2009). https://doi.org/10.1016/j.biosystems.2009.07.007

7. Dorier, J., Crespo, I., Niknejad, A., Liechti, R., Ebeling, M., Xenarios, I.: Boolean regulatory network reconstruction using literature based knowledge with a genetic algorithm optimization method. BMC Bioinformatics 17(1), 410 (2016). https://doi.org/10.1186/s12859-016-1287-z

8. Eiter, T., Gottlob, G.: On the computational cost of disjunctive logic programming: Propositional case. Annals of Mathematics and Artificial Intelligence 15(3), 289323 (Sep 1995). https://doi.org/10.1007/BF01536399

9. Eiter, T., Ianni, G., Krennwallner, T.: Answer Set Programming: A Primer, pp. 40-110. Springer Berlin Heidelberg, Berlin, Heidelberg (2009). https://doi.org/10.1007/978-3-642-03754-2_2

10. Gebser, M., Kaminski, R., Kaufmann, B., Schaub, T.: Answer Set Solving in Practice. Synthesis Lectures on Artificial Intelligence and Machine Learning, Morgan and Claypool Publishers (2012)

11. Gebser, M., Kaminski, R., Kaufmann, B., Schaub, T.: Clingo = ASP + control: Preliminary report. CoRR abs/1405.3694 (2014)

12. Gillespie, D.T.: A general method for numerically simulating the stochastic time evolution of coupled chemical reactions. Journal of computational physics 22(4), 403-434 (1976). https://doi.org/10.1016/0021-9991(76)90041-3

13. Goldfeder, J., Kugler, H.: BRE:IN - a backend for reasoning about interaction networks with temporal logic. In: Computational Methods in Systems Biology, pp. 289-295. Springer International Publishing (2019). https://doi.org/10.1007/978-3030-31304-3_15

14. Kauffman, S.: A proposal for using the ensemble approach to understand genetic regulatory networks. Journal of Theoretical Biology 230(4), 581-590 (oct 2004). https://doi.org/10.1016/j.jtbi.2003.12.017

15. Klarner, H., Bockmayr, A., Siebert, H.: Computing maximal and minimal trap spaces of boolean networks. Natural Computing 14(4), 535-544 (2015). https://doi.org/10.1007/s11047-015-9520-7 
16. Krawitz, P., Shmulevich, I.: Basin entropy in boolean network ensembles. Physical Review Letters 98(15) (apr 2007). https://doi.org/10.1103/physrevlett.98.158701

17. Lin, F., Zhao, Y.: ASSAT: Computing answer sets of a logic program by SAT solvers. Artificial Intelligence 157(1), 115-137 (2004). https://doi.org/10.1016/j.artint.2004.04.004

18. Lobo, J., Minker, J., Rajasekar, A.: Foundations of disjunctive logic programming. MIT press (1992)

19. Paulevé, L., Kolčák, J., Chatain, T., Haar, S.: Reconciling qualitative, abstract, and scalable modeling of biological networks. bioRxiv (2020). https://doi.org/10.1101/2020.03.22.998377

20. Razzaq, M., Kaminski, R., Romero, J., Schaub, T., Bourdon, J., Guziolowski, C.: Computing diverse boolean networks from phosphoproteomic time series data. In: Computational Methods in Systems Biology, pp. 59-74. Springer International Publishing (2018). https://doi.org/10.1007/978-3-319-99429-1_4

21. Schwieger, R., Siebert, H.: Graph representations of monotonic boolean model pools. In: Computational Methods in Systems Biology, pp. 233-248. Springer International Publishing (2017). https://doi.org/10.1007/978-3-319-67471-1_14

22. Stoll, G., Caron, B., Viara, E., Dugourd, A., Zinovyev, A., Naldi, A., Kroemer, G., Barillot, E., Calzone, L.: MaBoSS 2.0: an environment for stochastic boolean modeling. Bioinformatics 33(14), 2226-2228 (2017). https://doi.org/10.1093/bioinformatics/btx123

23. Stoll, G., Viara, E., Barillot, E., Calzone, L.: Continuous time boolean modeling for biological signaling: application of gillespie algorithm. BMC systems biology 6(1), 116 (2012). https://doi.org/10.1186/1752-0509-6-116

24. Terfve, C., Cokelaer, T., Henriques, D., MacNamara, A., Goncalves, E., Morris, M.K., Iersel, M.v., Lauffenburger, D.A., Saez-Rodriguez, J.: CellNOptR: a flexible toolkit to train protein signaling networks to data using multiple logic formalisms. BMC Systems Biology 6(1), 133 (2012). https://doi.org/10.1186/1752-0509-6-133

25. Van Kampen, N.G.: Stochastic processes in physics and chemistry, vol. 1. Elsevier (1992)

26. Wold, S., Esbensen, K., Geladi, P.: Principal component analysis. Chemometrics and intelligent laboratory systems 2(1-3), 37-52 (1987). https://doi.org/10.1016/0169-7439(87)80084-9

27. Yordanov, B., Dunn, S.J., Kugler, H., Smith, A., Martello, G., Emmott, S.: A method to identify and analyze biological programs through automated reasoning. Systems Biology and Applications 2 (2016). https://doi.org/10.1038/npjsba.2016.10

28. Zañudo, J.G., Steinway, S.N., Albert, R.: Discrete dynamic network modeling of oncogenic signaling: Mechanistic insights for personalized treatment of cancer. Current Opinion in Systems Biology 9, 1-10 (2018). https://doi.org/10.1016/j.coisb.2018.02.002 